\title{
Signature of the Van der Waals like small-large charged AdS black hole phase transition in quasinormal modes
}

\section{Yunqi Liu, De-Cheng Zou, and Bin Wang}

Department of Physics and Astronomy, Shanghai Jiao Tong University, Shanghai 200240, China

E-mail: liuyunqi@sjtu.edu.cn, zoudecheng@sjtu.edu.cn, wang_b@sjtu.edu.cn

ABSTRACT: We calculate the quasinormal modes of massless scalar perturbations around small and large four-dimensional Reissner-Nordstrom-Anti de Sitter (RN-AdS) black holes. We find a dramatic change in the slopes of quasinormal frequencies in small and large black holes near the critical point where the Van der Waals like thermodynamic phase transition happens. This further supports that the quasinormal mode can be a dynamic probe of the thermodynamic phase transition.

KeYwords: Black Holes, AdS-CFT Correspondence

ArXiv EPRINT: 1405.2644 


\section{Contents}

1 Introduction 1

2 Phase transition in charged AdS black hole spacetime 2

3 Perturbations in the charged AdS black hole spacetime 4

3.1 Isobaric phase transition 4

$\begin{array}{lll}3.2 & \text { Isothermal phase transition } & 7\end{array}$

4 The behavior of quasinormal frequencies at the critical point 12

5 Conclusion $\quad 14$

\section{Introduction}

Black hole thermodynamics has been an intriguing subject of discussions for decades. Inspired by the AdS/CFT correspondence, the black hole thermodynamics in the presence of a negative cosmological constant has become even more appealing. The thermodynamic property of AdS black holes was first investigated in [1] where it was found that there exists a Hawking-Page phase transition between the Schwarzschild AdS black hole and pure AdS space. Later it was further disclosed that in the charged AdS black holes there is a first order phase transition between small and large black holes in the canonical ensemble $[2,3]$. This phase transition was argued superficially analogous to a liquid-gas phase transition in the Van der Waals fluid. The superficial reminiscence was also observed in other AdS black hole backgrounds [4-17]. Recently the study of thermodynamics in AdS black holes has been generalized to the extended phase space, where the cosmological constant is identified with thermodynamic pressure, $P=-\frac{\Lambda}{8 \pi}=\frac{3}{8 \pi l^{2}}$, in the geometric units $G_{N}=\hbar=c=k=1 . l$ denotes the AdS radius, which is considered varying [18-22] and is included in the first law of black hole thermodynamics to ensure the consistency between the first law of black hole thermodynamics and the Smarr formula [23]. With the varying cosmological constant, the AdS black hole mass is identified with enthalpy and there exists a natural conjugate thermodynamic volume to the cosmological constant. In the extended phase space with cosmological constant and volume as thermodynamic variables, it was interestingly observed that the small-large black hole system admits a more direct and precise coincidence with the Van der Waals system [24]. More discussions on comparing phase transitions in AdS black holes with the Van der Waals analogy can be found in [25-39].

It has been an expectation for a long time that black hole thermodynamical phase transitions can have some observational signatures to be detected. Considering that quasinormal modes of dynamical perturbations are characteristic sounds of black holes [40-43], 
it is expected that black hole phase transitions can be reflected in the dynamical perturbations in the surrounding geometries of black holes through frequencies and damping times of the oscillations.

In asymptotically flat Reissner-Nordstrom $(\mathrm{RN})$ black hole, it was argued that a second order phase transition happens where the heat capacity appears singular [44, 45]. This thermodynamical second order phase transition point was disclosed in the dynamical quasinormal modes [46]. The observed relation between thermodynamical phase transitions and dynamical perturbations is not trivial [47] and was also confirmed in [48]. In AdS black holes, thermodynamic phase transition in the dual field theory corresponds to the onset of instability of a black hole, so that quasinormal modes of black holes are naturally connected with thermodynamic phase transitions of strongly coupled field theories [49]. Besides, the second-order phase transition of a topological AdS black hole to a hairy configuration was found reflected in the quasinormal modes of the electromagnetic and scalar perturbations, respectively [50, 51]. Moreover the phase transition in the charged topological-AdS black holes was observed in the quasinormal modes of the electromagnetic and gravitational perturbations [52]. Different phase properties between the massless BTZ black hole and the generic nonrotating BTZ hole were also detected in the scalar field as well as the fermonic field perturbations [53]. Phase transition between scalar and non-rotating BTZ black holes in three dimensions was also shown in the dynamical perturbation behaviors [54]. In [55], it was even argued that the thermodynamical stability is closely related to the dynamical stability for black brane solutions. More recently, the phase transitions before and after the scalar field condensation in the backgrounds of the AdS black hole and AdS soliton were further observed in the quasinormal modes of dynamical perturbations [56-59].

It is of great interest to generalize the discussions on the relation between thermodynamical phase transitions and dynamical perturbations to the Van der Waals like phase transitions in RN-AdS black holes. In this paper we will concentrate on this topic and disclose the fact that quasinormal modes can again be a probe of the phase transition in the Van der Waals analogy system. In section 2 we will first review the analogy of the small-large black hole system with the Van der Waals system in the extended phase space. In section 3, we will disclose numerically that different phases can be reflected by the quasinormal modes of dynamical perturbations. We will summarize our results in the last section.

\section{Phase transition in charged AdS black hole spacetime}

We consider a four-dimensional RN-AdS black hole with the metric

$$
\begin{aligned}
& d s^{2}=-f(r) d t^{2}+\frac{1}{f(r)} d r^{2}+r^{2} d \Omega_{2}^{2}, \\
& f(r)=1+\frac{r^{2}}{l^{2}}-\frac{2 M}{r}+\frac{Q^{2}}{r^{2}}
\end{aligned}
$$

where $M$ and $Q$ are the mass and charge of the black hole. In terms of the black hole event horizon $r_{H}$, the mass $M$, Hawking temperature $T$, entropy $S$ and electromagnetic 
potential $\Phi$ of the RN-AdS black hole can be expressed as

$$
\begin{aligned}
M & =\frac{r_{H}}{2}\left(1+\frac{r_{H}^{2}}{l^{2}}+\frac{Q^{2}}{r_{H}^{2}}\right), & T & =\frac{1}{4 \pi r_{H}}\left(1+\frac{3 r_{H}^{2}}{l^{2}}-\frac{Q^{2}}{r_{H}^{2}}\right), \\
S & =\pi r_{H}^{2}, & \Phi & =\frac{Q}{r_{H}} .
\end{aligned}
$$

Considering the thermodynamic volume $V=4 \pi r_{H}^{3} / 3$ and the pressure $P=3 /\left(8 \pi l^{2}\right)$, we can have the first law of black hole thermodynamics in an extended phase space [24]

$$
d M=T d S+\Phi d Q+V d P
$$

where the black hole mass $M$ can be considered as the enthalpy rather than the internal energy of the gravitational system [23].

In addition, the expression of the black hole temperature can be translated into the equation of state $P(V, T)$

$$
P=\frac{T}{2 r_{H}}-\frac{1}{8 \pi r_{H}^{2}}+\frac{Q^{2}}{8 \pi r_{H}^{4}}, \quad r_{H}=(3 V / 4 \pi)^{1 / 3} .
$$

Then the critical point can be obtained from

$$
\left.\frac{\partial P}{\partial r_{H}}\right|_{T=T_{c}, r_{H}=r_{c}}=\left.\frac{\partial^{2} P}{\partial r_{H}^{2}}\right|_{T=T_{c}, r_{H}=r_{c}}=0,
$$

which leads to $T_{c}=\frac{\sqrt{6}}{18 \pi Q}, r_{c}=\sqrt{6} Q$ and $P_{c}=\frac{1}{96 \pi Q^{2}}$.

In the extended phase space, the Gibbs free energy for fixed charge is read

$$
G(T, P)=\frac{1}{4}\left[r_{H}-\frac{8 \pi P r_{H}^{3}}{3}-\frac{3 Q^{2}}{r_{H}}\right] .
$$

Here $G$ is understood as a function of pressure and temperature by considering the equation of state eq. (2.5).

Figure 1 shows the Gibbs free energy and temperature coincide for small and large black holes [24]. The curve is the coexistence line of small-large black hole phase transition of the charged AdS black hole system. The critical point is highlighted by a small circle at the end of the coexistence line. From figure 1 we can see that to accommodate the phase transition from small to large black holes, we can choose either the isobaric or isothermal process. Then, we display the behaviors of the Gibbs free energy in the isobaric process by fixing $P$ in figure 2 and the Gibbs free energy in the isothermal process by fixing the temperature of the system in figure 3, respectively. It is clear that both $G$ surfaces demonstrate the characteristic swallow tail behavior, marking the first order transition in the system. In addition, the corresponding $T-r_{H}$ and $P-r_{H}$ diagrams of charged AdS black holes for the isobaric and isothermal processes are also shown in figure 2 and figure 3 , respectively. Both of them contain inflection points and the behaviors are reminiscent of Van der Waals system [24]. 


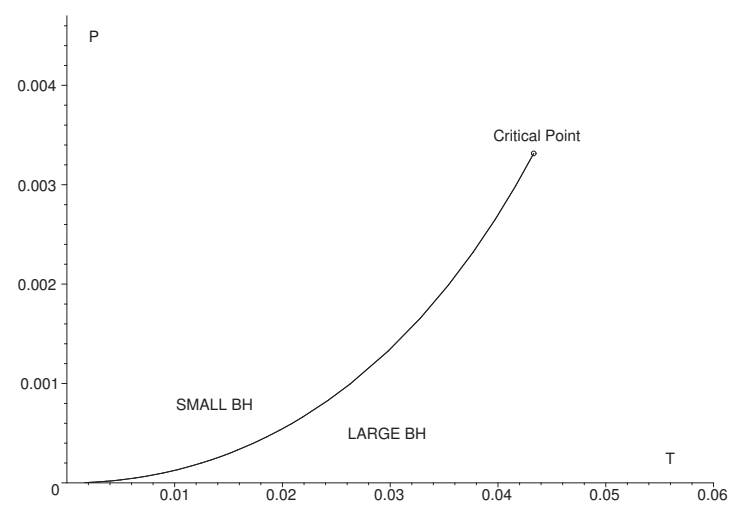

Figure 1. The coexistence line of charged AdS black hole of small-large black hole phase transition in P-T plane with a unit black hole charge.

\section{Perturbations in the charged AdS black hole spacetime}

Now we consider a massless scalar field perturbation in the surrounding geometry of the four-dimensional RN-AdS black hole background eq. (2.1). The radial part of the perturbation, $\Psi(r, t)=\psi(r) e^{-i \omega t}$, is described by the Klein-Gordon equation

$$
\psi^{\prime \prime}(r)+\left[\frac{f^{\prime}(r)}{f(r)}+\frac{2}{r}\right] \psi^{\prime}(r)+\frac{w^{2} \psi(r)}{f(r)^{2}}=0,
$$

where $\omega$ indicates the frequency of the perturbation.

Near the black hole horizon $r_{H}$, we can impose the ingoing boundary condition, $\psi(r) \sim$ $\left(r-r_{H}\right)^{-i \frac{\omega}{4 \pi T}}$. Defining $\psi(r)$ as $\psi(r) \exp \left[-i \int \frac{\omega}{f(r)} d r\right]$, where $\exp \left[-i \int \frac{\omega}{f(r)} d r\right]$ asymptotically approaches the ingoing wave near horizon, we can rewrite eq. (3.1) into

$$
\psi^{\prime \prime}(r)+\psi^{\prime}(r)\left[\frac{f^{\prime}(r)}{f(r)}-\frac{2 i \omega}{f(r)}+\frac{2}{r}\right]-\psi(r) \frac{2 i \omega}{r f(r)}=0,
$$

so that when $r \rightarrow r_{H}$, we can set $\psi(r)=1$. At the AdS boundary $r \rightarrow \infty$, we need $\psi(r)=0$. With the boundary conditions we solve eq. (3.2) numerically and find the frequencies of the quasinormal modes by using the shooting method.

We are going to study whether the signature of thermodynamical first order phase transition in charged AdS black holes, in analogy to the liquid-gas phase transition, can be reflected in the dynamical quasinormal modes behavior in the massless scalar perturbation. We will examine the dynamical perturbations in the possible two processes, namely isobaric process by fixing the pressure $P$ and the isothermal process by fixing the temperature $T$, to accommodate the phase transitions between small-large black holes. We will set the black hole charge to be unity in our following numerical computations.

\subsection{Isobaric phase transition}

In this case, $P$ (or $l$ ) is fixed so that the black hole horizon $r_{H}$ is the only variable in the system. We have seen the behavior of isobar in the $T-r_{H}$ diagram in figure 2 . The oscillating part is for $P<P_{c}$, where a small-large black hole phase transition occurs in 

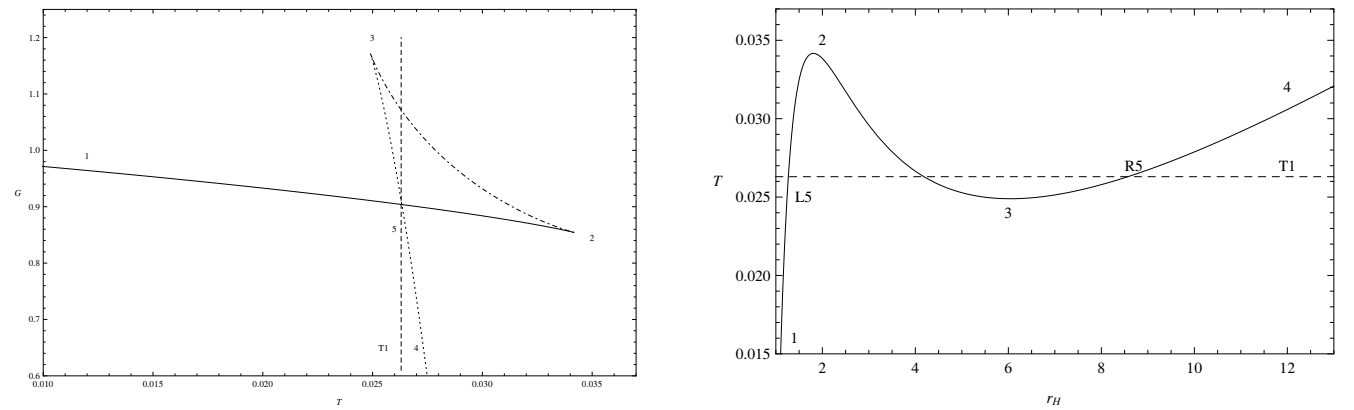

Figure 2. Left: the Gibbs free energy is given as a function of black hole temperature for $P=0.001$. Solid line(Line1-2) and dotted line(Line3-4) cross at the point "5", indicating the place where the phase transition happens. Right: the black hole temperature $T$ is depicted as a function of the black hole horizon $r_{H}$ for $P=0.001$. The dashed line indicates the phase transition temperature.
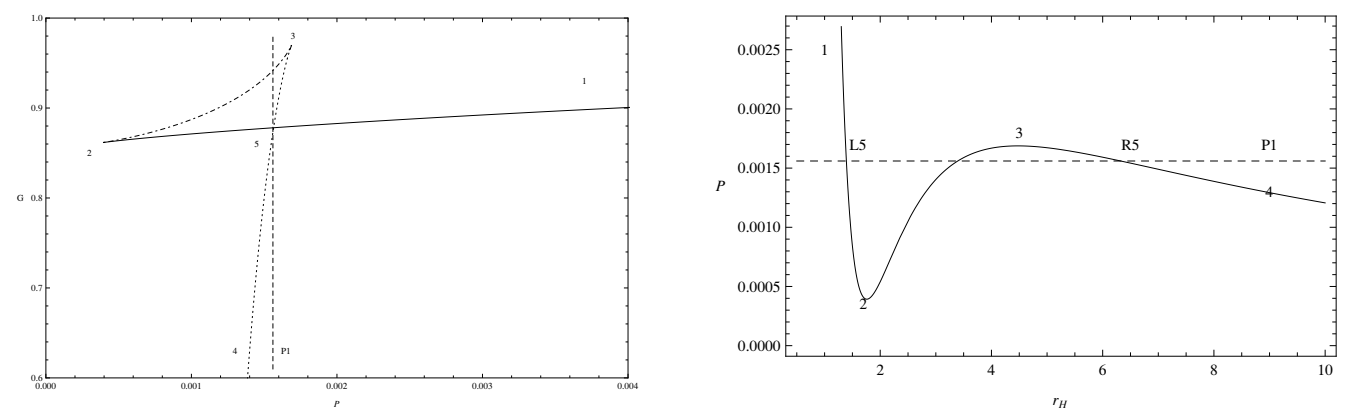

Figure 3. Left: the Gibbs free energy is given as a function of pressure $P$ for the isothermal phase transition with $T=0.0032$. Solid line 1-2 and dotted line 3-4 cross each other at the point "5", which indicates the phase transition point. Right: the black hole pressure $P$ is depicted as a function of black hole horizon $r_{H}$ with $T=0.0032$. The dashed line indicates the phase transition pressure.

the system. The critical isobar $P_{c}$ is got by $\frac{\partial T}{\partial r_{H}}=\frac{\partial^{2} T}{\partial r_{H}^{2}}=0$. More information of the phase transition is reflected in the Gibbs free energy in the left panel of figure 2. The solid line and the dotted line cross each other at the intersection point marked as "5", which indicates the coexistence of two phases in equilibrium. In the right panel this point is separated into "L5" and "R5", which have the same Gibbs free energy and the same black hole temperature $T=T_{c} \simeq 0.02630$ for small and large black holes. Combining the Gibbs free energy and the phase diagram, we find that the physical phase marked between points "1-5" or "1-L5" is for the small black hole, while physical phase indicated between points "5-4" or "R5-4" is for the large black hole.

In table 1, we list the frequencies of the quasinormal modes of the massless scalar perturbation around small and large black holes described between points "1-L5" and "R54" respectively in figure 2 for different temperatures. The frequencies above the horizontal line are for the small black hole phase while the frequencies below are for the large black hole phase.

For the small black hole phase, we see that when the temperature decreases from the phase transition critical point $T_{c}$, the black hole becomes smaller. In this process the real part frequencies change very little, while the absolute values of the imaginary part 


\begin{tabular}{|c|c|c|}
\hline$T\left(10^{-2}\right)$ & $r_{H}$ & $\omega$ \\
\hline 2.2 & 1.190 & $0.2223-0.02397 \mathrm{I}$ \\
2.3 & 1.206 & $0.2222-0.02408 \mathrm{I}$ \\
2.4 & 1.223 & $0.2222-0.02421 \mathrm{I}$ \\
2.5 & 1.242 & $0.2221-0.02436 \mathrm{I}$ \\
2.55 & 1.252 & $0.2221-0.02445 \mathrm{I}$ \\
2.6 & 1.262 & $0.2220-0.02454 \mathrm{I}$ \\
\hline 2.65 & 8.774 & $0.2364-0.1963 \mathrm{I}$ \\
2.7 & 9.248 & $0.2403-0.2070 \mathrm{I}$ \\
2.8 & 10.099 & $0.2478-0.2262 \mathrm{I}$ \\
2.9 & 10.871 & $0.2551-0.2436 \mathrm{I}$ \\
3.1 & 12.282 & $0.2695-0.2752 \mathrm{I}$ \\
\hline
\end{tabular}

Table 1. The quasinormal frequencies of massless scalar perturbation with the change of black hole temperature. The upper part, above the horizontal line, is for the small black hole phase, while the lower part is for the large black hole phase.

of quasinormal frequencies decrease. This result is consistent with the objective picture obtained in [60]. Considering that the decay of the test field outside the black hole is due to the black hole absorption, it is natural to understand that when the black hole becomes much smaller, its absorption ability decreases so that the field will decay slower and the oscillation (real part of the perturbation) nearly keeps as a constant.

For the large black hole phase, we find that when the temperature increases from the critical value $T_{c}$, the black hole gets bigger. The real part together with the absolute value of the imaginary part of quasinormal frequencies increase. The massless scalar perturbation outside the black hole gets more oscillations but it decays faster. To have a physical picture of this result, we plot the effective potential behavior near the AdS boundary. In the AdS boundary, when $r \rightarrow \infty$, the tortoise coordinate $r_{\text {AdS }}^{*}$ tends to a constant value [61]. With the increase of $r_{H}, r_{\text {AdS }}^{*}$ at the AdS boundary becomes smaller. Thus the infinite potential wall at the AdS boundary will be moved towards the black hole. This will bounce back more outgoing perturbation towards the black hole, which will add energy to the perturbation and make the real part frequency increase. On the other hand, when the black hole becomes bigger, it becomes more greedy and can absorb more things. This can explain that the absolute value of the imaginary part increases with the black hole size, so that the decay of the perturbation becomes faster.

The drastically different quasinormal frequencies for small and large black hole phases are plotted in figure 4. In the left panel, to plot the figure we took the value of the real part as five significant digits while it was taken as four in table 1, actually the real part varies little here. From the figure, we can see different slopes of the quasinormal frequencies in the massless scalar perturbations reveal that small and large black holes are in different phases. To change the small black hole to be a large one, one has to encounter a phase transition. The different properties disclosed here in dynamical perturbations reflect the idea of the thermodynamic phase transitions between small-large RN-AdS black holes. 

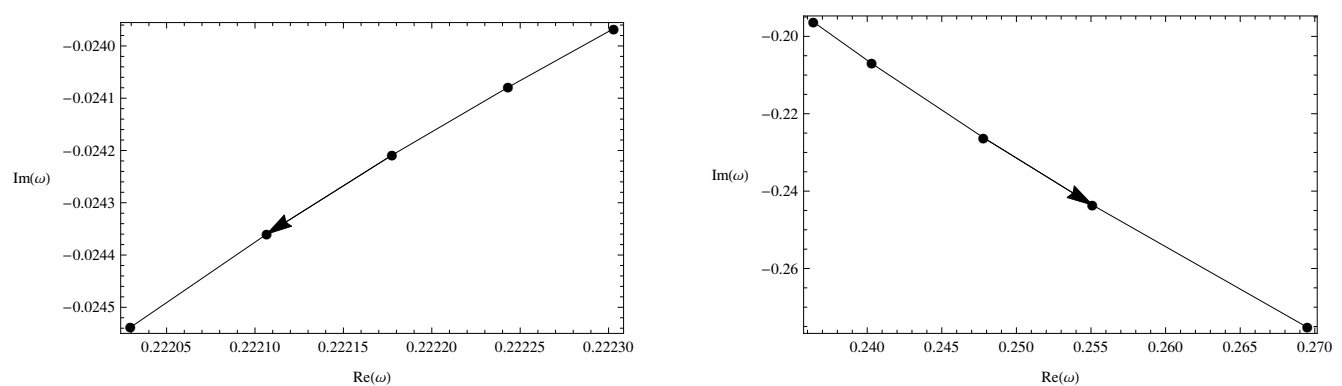

Figure 4. The left panel shows the behavior of quasinormal modes for small black holes and the right panel shows the behavior of quasinormal modes for large black holes. The arrow indicates the increase of black hole horizon.

\subsection{Isothermal phase transition}

Fixing the black hole temperature $T$, we can plot the $P-r_{H}$ diagram of charged AdS black holes in the right panel of figure 3. For $T<T_{c}$ there is an inflection point and the behavior is reminiscent of the Van der Waals system. The critical point can be got from $\frac{\partial P}{\partial r_{H}}=\frac{\partial^{2} P}{\partial r_{H}^{2}}=0$. The behavior of the Gibbs free energy is plotted in the left panel of figure 3. The cross point " 5 " between the solid line marked as " $1-5$ " and the dotted line denoted as "4-5" shows that the Gibbs free energy and $P$ coincide for small and large black holes. In the right panel of the phase diagram, the point " 5 " is separated into "L5" and "R5" for the same Gibbs free energy and the chosen $P \simeq 0.00156$ where the small and large black hole can coexist. Combining the Gibbs free energy and the phase diagram, we find that the physical phase marked between points "1-5" or "1-L5" is for the small black hole, while physical phase indicated between points " $5-4$ " or "R5-4" is for the large black hole.

In table 2 we list the frequencies of quasinormal modes for small and large black hole phases in the isothermal phase transition with $T=0.032$. The data above the horizontal line are the frequencies for small black holes, while those below are for large black holes. We can see that for the small black hole phase, as the black hole horizon grows, the corresponding pressure $P$ decreases. In this process the real parts of the frequencies decrease and the absolute imaginary parts decrease as well. For the large black hole case, with the increase of the black hole size, the pressure decreases. In this process, the real parts of the quasinormal frequencies decrease while the absolute values of the imaginary parts increase. Figure 5 shows the behaviors of the quasinormal modes for small and large holes. The arrows indicate the increase of the black hole size. It is clear that in small and large black hole phases, the properties of the quasinormal frequencies are completely different.

In the isothermal phase transition, besides the variable $r_{H}$, the AdS radius $l$ is another variable to influence the quasinormal behaviors. They are related by the fixed temperature, however each of them has independent influence on the quasinormal modes. Now let's first examine the independent influence on the quasinormal frequencies by each of these two parameters. By fixing $l(P)$, we list the influence of $r_{H}$ on the frequencies for small and large black holes in table 3 and 4 . As the physical interpretation given above, for the small black hole phase the quasinormal modes exhibits a nearly constant oscillation and decays slower when the black hole further shrinks. For the large black holes when $r_{H}$ increases, 


\begin{tabular}{|c|c|c|}
\hline$P\left(10^{-3}\right)$ & $r_{H}$ & $\omega$ \\
\hline 2.0 & 1.350 & $0.2955-0.06489 \mathrm{I}$ \\
1.9 & 1.359 & $0.2892-0.06100 \mathrm{I}$ \\
1.8 & 1.368 & $0.2827-0.05714 \mathrm{I}$ \\
1.7 & 1.377 & $0.2761-0.05328 \mathrm{I}$ \\
1.6 & 1.387 & $0.2692-0.04944 \mathrm{I}$ \\
\hline 1.5 & 6.906 & $0.2860-0.2320 \mathrm{I}$ \\
1.45 & 7.389 & $0.2854-0.2400 \mathrm{I}$ \\
1.4 & 7.880 & $0.2848-0.2472 \mathrm{I}$ \\
1.35 & 8.388 & $0.2840-0.2538 \mathrm{I}$ \\
1.3 & 8.919 & $0.2831-0.2599 \mathrm{I}$ \\
1.25 & 9.480 & $0.2822-0.2656 \mathrm{I}$ \\
1.2 & 10.075 & $0.2811-0.2710 \mathrm{I}$ \\
1.1 & 11.396 & $0.2790-0.2809 \mathrm{I}$ \\
\hline
\end{tabular}

Table 2. The quasinormal frequencies of massless scalar perturbations for black holes with different sizes in the isothermal phase transition with $T=0.032$. The upper part, above the horizontal line, is the frequency for the small black hole phase, while the lower part is for the large black hole phase.
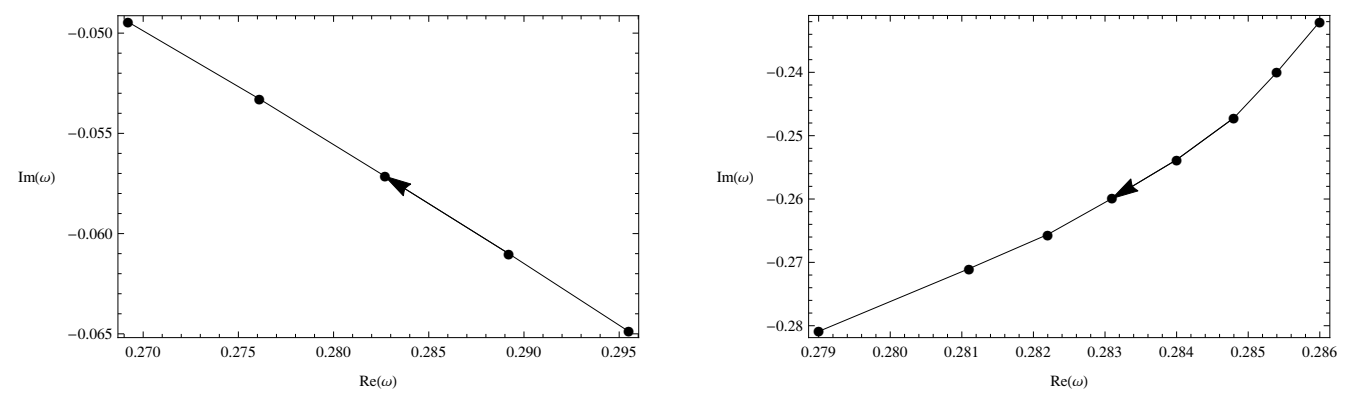

Figure 5. The left panel shows the behavior of quasinormal modes for small black holes and the right panel exhibits the behavior of quasinormal modes for large black holes. The arrows in the figure indicate the increase of the black hole size.

as shown in figure 6 the AdS wall moves closer to the black hole which will bounce back the outgoing perturbations more strongly to increase the real part of the quasinormal frequency. At the meantime, the black hole becomes bigger to swallow more things, which results in the fast decay of the perturbation.

Now let's turn to discuss the independent effect of $l(P)$ on the quasinormal frequency by fixing the black hole size $r_{H}$. For small and large black hole phases, the results are listed in table 5 and 6 . For both small and large holes, with the decrease of $P$ (the increase of $l$ ), both the real parts and the absolute imaginary parts of quasinormal frequencies decrease. The effects of increasing $l$ on the quasinormal frequency for small and large holes are completely different from the effect of increasing the black hole size $r_{H}$. This can be 


\begin{tabular}{|c|c|c|}
\hline$P\left(10^{-3}\right)$ & $r_{H}$ & $\omega$ \\
\hline 2.0 & 1.35 & $0.29547-0.06489 \mathrm{I}$ \\
\hline 2.0 & 1.40 & $0.29542-0.06604 \mathrm{I}$ \\
\hline 2.0 & 1.45 & $0.29540-0.06734 \mathrm{I}$ \\
\hline 2.0 & 1.50 & $0.29540-0.06875 \mathrm{I}$ \\
\hline
\end{tabular}

Table 3. This table shows how the quasinormal frequencies change as the black hole horizons $r_{H}$ increase for small black holes. The pressure is fixed as $P=0.002$.

\begin{tabular}{|c|c|c|}
\hline$P\left(10^{-3}\right)$ & $r_{H}$ & $\omega$ \\
\hline 1.4 & 7.70 & $0.2826-0.2415 \mathrm{I}$ \\
\hline 1.4 & 7.88 & $0.2848-0.2472 \mathrm{I}$ \\
\hline 1.4 & 8.00 & $0.2862-0.2510 \mathrm{I}$ \\
\hline
\end{tabular}

Table 4. This table shows how the quasinormal frequencies change as black hole horizons $r_{H}$ increase for large black holes. The pressure is fixed as $P=0.0012$.

attributed to different boundary behaviors caused by the increases of $l$ and $r_{H}$. With the increase of $r_{H}$, we saw in figure 6 that the AdS wall moves closer to the black hole. But when the AdS radius $l$ increases, the AdS wall moves further away, which is illustrated in figure 7 . This results in the mildly bouncing back of the outgoing perturbations when $l$ increases compared with the increase of $r_{H}$, which explains the decrease of the real part of the frequency. But the outgoing perturbation will be mildly bounced back continuously with the increase of $l$, while the black hole size is fixed so that the amount of perturbation it can swallow is fixed. This accounts for the physical reason that the decay becomes slower when $l$ increases.

Thus we see that the influences given by $r_{H}$ and $l$ on quasinormal frequencies of the perturbation are different. The competition between these two factors result in the properties of the quasinormal frequencies for small and large black holes shown in table 2 and figure 5 .

To see closely how these two factors compete with each other to influence the quasinormal frequencies, we do a double-series expansion of the frequency $\omega\left(r_{H}+\triangle r_{H}, P+\triangle P\right)$ in $\left(\triangle r_{H}, \triangle P\right)$,

$$
\begin{aligned}
\omega\left(r_{H}+\right. & \left.\triangle r_{H}, P+\triangle P\right) \\
& =\omega\left(r_{H}, P\right)+\partial_{r_{H}} \omega \triangle r_{H}+\partial_{P} \omega \triangle P+O\left(\triangle r_{H}^{2}, \triangle P^{2}, \triangle r_{H} \triangle P\right) .
\end{aligned}
$$

This means that the changes of the quasinormal frequency get two influences, one is from the change of the black hole size $r_{H}$, and the other is from the change of the pressure $P$ (or AdS radius $l$ ). For simple discussions in the following, we define $\Delta_{1} \equiv \partial_{r_{H}} \omega \Delta r_{H}$ and $\Delta_{2} \equiv \partial_{P} \omega \triangle P$. 


\begin{tabular}{|c|c|c|}
\hline$r_{H}$ & $P\left(10^{-3}\right)$ & $\omega$ \\
\hline 1.35 & 1.8 & $0.2827-0.0578 \mathrm{I}$ \\
\hline 1.35 & 1.9 & $0.2892-0.0608 \mathrm{I}$ \\
\hline 1.35 & 2.0 & $0.2955-0.0649 \mathrm{I}$ \\
\hline 1.35 & 2.1 & $0.3016-0.0690 \mathrm{I}$ \\
\hline
\end{tabular}

Table 5. This table shows how the quasinormal frequencies change as the pressure $P$ increases for small black holes, where the black hole horizon is fixed as $r_{H}=1.4$.

\begin{tabular}{|c|c|c|}
\hline$r_{H}$ & $P$ & $\omega$ \\
\hline 7.880 & 0.0013 & $0.2712-0.2294 \mathrm{I}$ \\
\hline 7.880 & 0.0014 & $0.2848-0.2472 \mathrm{I}$ \\
\hline 7.880 & 0.0015 & $0.2982-0.2650 \mathrm{I}$ \\
\hline
\end{tabular}

Table 6. This table shows how the quasinormal frequencies change as the pressure $P$ increases for large black holes, where the black hole horizon is fixed as $r_{H}=11$.

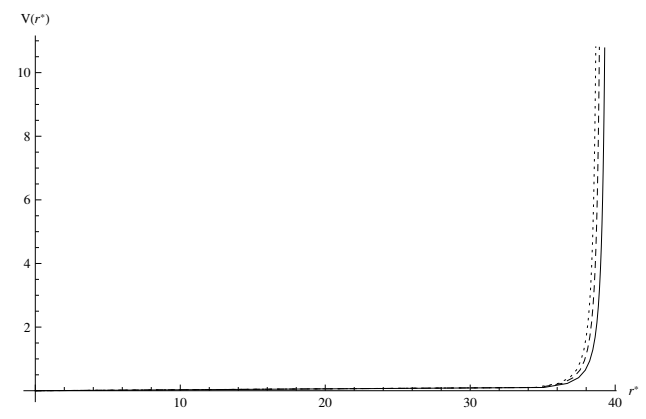

Figure 6. The potential is depicted for the fixed pressure $P=0.0014$ (or the fixed AdS radius $l$ ). At the AdS boundary, $r^{*}$ approaches a constant. With the increase of the black hole size (the solid line is for $r_{H}=7.7$, the dashed is for $r_{H}=7.88$ and the dotted line for $r_{H}=8$ ), the constant of $r^{*}$ decreases, so that the potential wall moves towards the black hole.

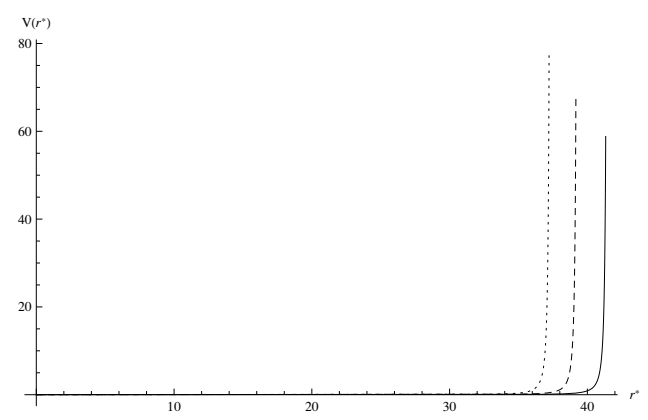

Figure 7. The potential is depicted for the fixed black hole size $r_{H}=7.88$, but different pressure $P$ (or AdS radius $l$ ). At the AdS boundary, $r^{*}$ approaches a constant. With the increase of the pressure $P$ (or the decrease of AdS radius $l$ ), the potential wall moves towards the black hole. The solid line is for $P=0.0013$, the dashed line is for $P=0.0014$ and the dotted for $P=0.0015$. 


\begin{tabular}{|c|c|c|c|c|}
\hline$r_{H}$ & $P\left(10^{-3}\right)$ & $\tilde{\omega}$ & $\triangle_{1}$ & $\triangle_{2}$ \\
\hline 1.35 & 2.0 & $0.2955-0.06489 \mathrm{I}$ & 0 & 0 \\
\hline 1.359 & 1.9 & $0.2893-0.06100 \mathrm{I}$ & $0-0.00022 \mathrm{I}$ & $-0.0062+0.0041 \mathrm{I}$ \\
\hline 1.368 & 1.8 & $0.2831-0.05713 \mathrm{I}$ & $-0.000013-0.00044 \mathrm{I}$ & $-0.012+0.0082 \mathrm{I}$ \\
\hline 1.377 & 1.7 & $0.2769-0.05325 \mathrm{I}$ & $-0.000019-0.00066 \mathrm{I}$ & $-0.019+0.012 \mathrm{I}$ \\
\hline
\end{tabular}

Table 7. For the small black hole phase, $\tilde{\omega}$ is the quasinormal frequency from the linear approximation. $\triangle_{1}$ and $\triangle_{2}$ are contributions from changes of the black hole size and pressure, respectively.

Considering the equation of state eq. (2.5), the changes of $P$ and $r_{H}$ are not completely independent. They are related by

$$
d P=\left(\frac{1}{4 \pi r_{H}^{3}}-\frac{Q^{2}}{2 \pi r_{H}^{5}}-\frac{T}{2 r_{H}^{2}}\right) d r_{H}
$$

Thus it is not arbitrary to choose the step length of $\triangle p$, it should be chosen following the step length of $\triangle r_{H}$.

From the frequencies of small black holes shown in tables 3 and 4 , we can get the derivative of $\omega$ with respect to $r_{H}$ at $r_{H}=1.35$ and $P=0.0002,\left.\partial_{r_{H}} \omega\right|_{r_{H}=1.35, P=0.002} \simeq$ $-0.0007-0.0245 I$ and the derivative of $\omega$ with respect to $P,\left.\partial_{P} \omega\right|_{r_{H}=1.35, P=0.002} \simeq 62-41 I$. Employing eq. (3.3), we can estimate the quasinormal frequencies, $\tilde{\omega}$, for small black holes from analytic linear expansion. The analytically obtained $\tilde{\omega}$ are listed in table 7 , whose behaviors are in good agreement with the numerical computation results listed in table II with the increase of the black hole size and the decrease of the pressure for small black holes. Comparing $\triangle_{1}$ and $\triangle_{2}$ in table 7 , we see that for small black holes, the change of $P$ (or $l$ ) clearly wins over the change of the black hole size, which dominantly contributes to the behavior of quasinormal frequencies for small black hole phase.

For the large black hole phase, to keep the linear approximation, we consider the small change of the black hole size. Near $P=0.0014$ and $r_{H}=7.880$ we have the derivatives $\left.\partial_{r_{H}} \omega\right|_{P=0.0014, r_{H}=7.880} \simeq 0.012-0.032 I$ and $\left.\partial_{P} \omega\right|_{P=0.0014, r_{H}=7.880} \simeq 135-178 I$. Using eq. (3.3), we can estimate the quasinormal frequencies $\tilde{\omega}$ from the linear approximation for the large black hole phase as shown in table 8 . To keep the linear approximation valid in eq. (3.3), we just did the estimation in the narrow range of $r_{H}$. $\tilde{\omega}$ keeps the property of the quasinormal frequencies with the increase of the black hole size and the decrease of the pressure as listed in table 2 and figure 5 for large black hole phase. $\triangle_{1}$ and $\triangle_{2}$ mark the contributions from the change of the black hole size and the pressure, respectively. It is clear that for the large black hole case, the change of the horizon size contributes more to the imaginary part of the frequency change. Thus the perturbation decays faster following the increase of the black hole size and has little dependence on the change of the pressure or the AdS length. The contributions of $\triangle_{1}$ and $\triangle_{2}$ on the real part of the frequency are comparable. But the change of $P$ (or $l$ ) wins out a little. Thus with the decrease of the $P$, the perturbation oscillation becomes a bit quieter. 


\begin{tabular}{|c|c|c|c|c|}
\hline$r_{H}$ & $P\left(10^{-3}\right)$ & $\tilde{\omega}$ & $\triangle_{1}$ & $\triangle_{2}$ \\
\hline 7.389 & 1.45 & $0.2856-0.2405 \mathrm{I}$ & $-0.0059+0.016$ & $0.0068-0.0089 \mathrm{I}$ \\
\hline 7.880 & 1.4 & $0.2848-0.2472 \mathrm{I}$ & 0 & 0 \\
\hline 8.388 & 1.35 & $0.2841-0.2544 \mathrm{I}$ & $0.0061-0.016 \mathrm{I}$ & $-0.0068+0.0089 \mathrm{I}$ \\
\hline
\end{tabular}

Table 8. For the large black hole phase, $\tilde{\omega}$ is the quasinormal frequency from the linear approximation. $\triangle_{1}$ and $\triangle_{2}$ are contributions from the changes of the black hole size and pressure, respectively.

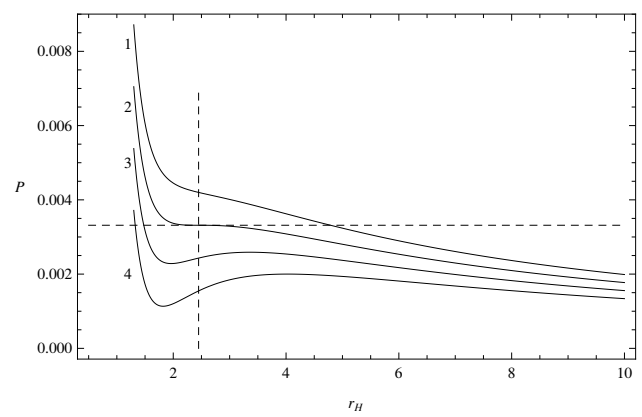

Figure 8. Lines marked 1-4 from the top to the bottom indicate the decrease of the temperature. Line 2 is for $T=T_{c}$. The cross of the dashed lines indicates the phase transition point of the critical isotherm state $T=T_{c}$.

We would like to point out that the quasinormal modes we considered are near the critical points of the phase transition. In the approximation we see that if the horizon $r_{H}$ becomes very big, eq. (3.4) will reduce to $d P=-\frac{T}{2 r_{H}^{2}} d r_{H}$, so that $\triangle P$ will be small enough and $\omega\left(r_{H}, P\right)$ will slowly vary with $P$, going back to the behavior in table 4 . Thus only near the critical point of the phase transition, we can have the behavior of slopes of quasinormal modes shown in figure 5, which is consistent with the description in the linear approximation.

\section{The behavior of quasinormal frequencies at the critical point}

For the isothermal phase transition, the critical point in figure 1 appears when the isotherm system starts to have an inflection point at $P=P\left(r_{H}\right)$, which is given by

$$
\left.\frac{\partial P}{\partial r_{H}}\right|_{T=T_{c}, r_{H}=r_{c}}=\left.\frac{\partial^{2} P}{\partial r_{H}^{2}}\right|_{T=T_{c}, r_{H}=r_{c}}=0
$$

where $T_{c}=\frac{\sqrt{6}}{18 \pi Q}, r_{c}=\sqrt{6} Q$ and $P_{c}=\frac{1}{96 \pi Q^{2}}$. At $T=T_{c}$, a second-order phase transition occurs [25]. Figure 8 shows the $P-r_{H}$ diagram for fixed temperature. The line 1 is for $T>T_{c}$, where there is no phase transition in the system. Line 2 marks the inflection point indicating the critical isotherm state $T=T_{c}$. Lines 3 and 4 are of $T<T_{c}$. The cross point of the dashed line shows the small-large black hole phase transition point at the $T=T_{c}$. Figure 9 depicts the free energy at $T=T_{c}$, the cross of the dashed lines indicates the phase 


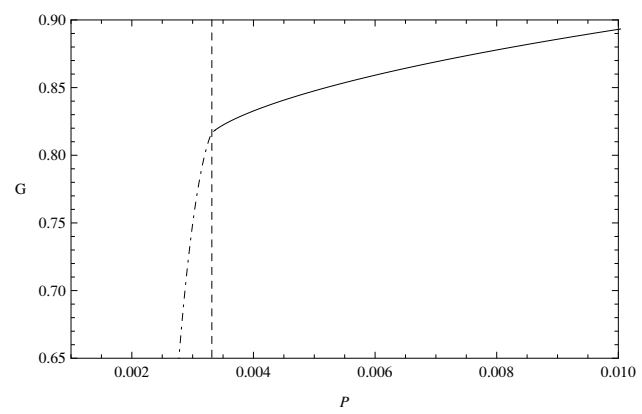

Figure 9. The free energy of the critical isotherm $T=T_{c}$. The dashed line indicates the phase transition point.

\begin{tabular}{|c|c|c|}
\hline$P\left(10^{-3}\right)$ & $r_{H}$ & $\omega$ \\
\hline 3.3160 & 2.376 & $0.4986-0.49766 \mathrm{I}$ \\
3.3158 & 2.401 & $0.4982-0.49768 \mathrm{I}$ \\
3.3157 & 2.425 & $0.4979-0.49770 \mathrm{I}$ \\
3.3157 & 2.449 & $0.4976-0.49773 \mathrm{I}$ \\
\hline 3.3157 & 2.474 & $0.4973-0.49776 \mathrm{I}$ \\
3.3157 & 2.498 & $0.4969-0.49780 \mathrm{I}$ \\
3.3153 & 2.572 & $0.4959-0.49794 \mathrm{I}$ \\
3.3125 & 2.694 & $0.4942-0.49826 \mathrm{I}$ \\
\hline
\end{tabular}

Table 9. The quasinormal frequencies of small and large black holes in the critical isothermal phase transition $T=T_{c}$. The data above the horizontal line are the frequencies for small black holes, while those below are for large black holes.

transition point. The free energy continuously varies from the small black hole phase to the large black hole phase which shows the character of a second-order phase transition. The quasinormal frequencies of massless scalar perturbation for different size black hole near the critical isothermal phase transition point with $T=T_{c}$ are given in table 9 . The data above the horizontal line are the frequencies for small black holes, while those below are for large black holes. The quasinormal frequencies for small and large black holes keep the same behavior as the black hole horizon increases.

For the isobaric phase transition $T=T\left(r_{H}\right)$, the phase transition begins to happen at the critical position,

$$
\left.\frac{\partial T}{\partial r_{H}}\right|_{P=P_{c}, r_{H}=r_{c}}=\left.\frac{\partial^{2} T}{\partial r_{H}^{2}}\right|_{P=P_{c}, r_{H}=r_{c}}=0 .
$$

Figure 10 depicts the $T-r_{H}$ diagram for fixed $P$. Lines from top to bottom correspond to the decrease of the pressure. Line 2 is for $P=P_{c}$. The cross of the dashed lines indicates the small-large black hole phase transition point in the isobaric phase transition at the critical point. The free energy at the critical point is depicted in figure 11, where the dashed line marks the transition point. Table 10 shows the quasinormal frequencies 


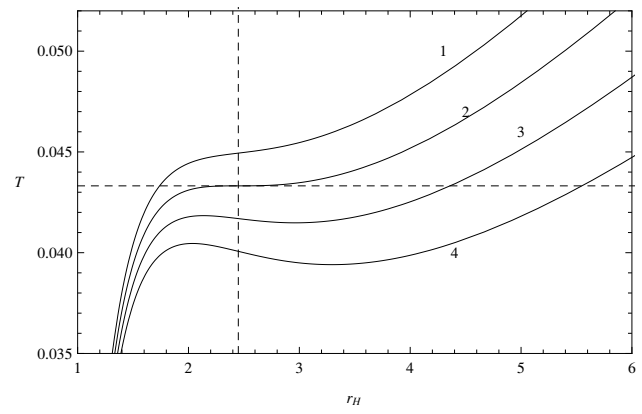

Figure 10. The lines from top to bottom are for different fixed pressure. Line 2 is for $P=P_{c}$. The cross point of the dashed lines indicates the phase transition point of the isobaric system at the critical point $P=P_{c}$.

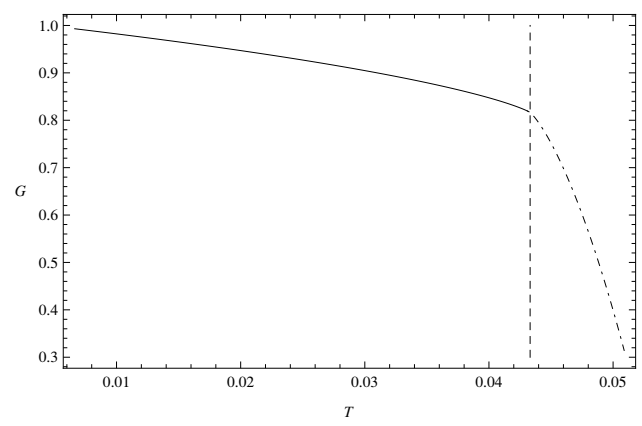

Figure 11. The free energy of the isobaric system at the critical point $P=P_{c}$. The dashed line indicates the phase transition point.

of the small and large black holes. The data above the horizontal line are the frequencies for small black holes, while those below are for large black holes. From the table we can see that at the critical point both the small and large black holes' quasinormal frequencies have the same behavior as the black hole horizon increases.

From both the critical isothermal and isobaric phase transitions, we learn that at the critical point the quasinormal frequencies keep the same behavior as the black hole horizon increases. Below these critical points $\left(P<P_{c}, T<T_{c}\right)$, quasinormal frequencies can reflect the phase transition between small and large black holes. Due to our numerical code efficiency, the difference in quasinormal frequencies can be clearer in a state much below the critical point.

\section{Conclusion}

We have calculated the quasinormal modes of massless scalar perturbations around small and large four-dimensional RN-AdS black holes. We found that when the Van der Waals analogy thermodynamic phase transition happens, no matter in the isobaric process by fixing the pressure in the extended space or in the isothermal process by fixing the temperature of the system, the slopes of the quasinormal frequency change drastically different in the small and large black holes as we increase the value of the horizon radius. This clearly presents the signature of the phase transition between small and large black holes. 


\begin{tabular}{|c|c|c|}
\hline$T\left(10^{-2}\right)$ & $r_{H}$ & $\omega$ \\
\hline 4.32 & 2.079 & $0.5006-0.4968 \mathrm{I}$ \\
4.32 & 2.103 & $0.5006-0.4969 \mathrm{I}$ \\
4.33 & 2.168 & $0.5005-0.4973 \mathrm{I}$ \\
4.33 & 2.449 & $0.4976-0.4977 \mathrm{I}$ \\
\hline 4.34 & 2.790 & $0.4933-0.4991 \mathrm{I}$ \\
4.34 & 2.889 & $0.4924-0.5000 \mathrm{I}$ \\
4.34 & 2.962 & $0.4919-0.5008 \mathrm{I}$ \\
4.35 & 3.022 & $0.4916-0.5016 \mathrm{I}$ \\
\hline
\end{tabular}

Table 10. The quasinormal frequencies of small and large black holes in the isobaric phase transition at $P=P_{c}$. The data above the horizontal line are the frequencies for small black holes, while those below are for large black holes.

This is one more example shows that the quasinormal mode can provide dynamical physical phenomenon of the thermodynamic phase transition. Since the quasinormal mode is expected to be detected and has strong astrophysical interest, its ability to reflect the thermodynamic phase transition is interesting, which is expected to disclose the observational signature of the thermodynamic phase transition.

\section{Acknowledgments}

This work was supported by the National Natural Science Foundation of China. Yunqi Liu was also supported by the China Postdoctoral Science Foundation.

Open Access. This article is distributed under the terms of the Creative Commons Attribution License (CC-BY 4.0), which permits any use, distribution and reproduction in any medium, provided the original author(s) and source are credited.

\section{References}

[1] S.W. Hawking and D.N. Page, Thermodynamics of black holes in Anti-de Sitter space, Commun. Math. Phys. 87 (1983) 577 [InSPIRE].

[2] A. Chamblin, R. Emparan, C.V. Johnson and R.C. Myers, Charged AdS black holes and catastrophic holography, Phys. Rev. D 60 (1999) 064018 [hep-th/9902170] [INSPIRE].

[3] A. Chamblin, R. Emparan, C.V. Johnson and R.C. Myers, Holography, thermodynamics and fluctuations of charged AdS black holes, Phys. Rev. D 60 (1999) 104026 [hep-th/9904197] [INSPIRE].

[4] C. Niu, Y. Tian and X.-N. Wu, Critical phenomena and thermodynamic geometry of RN-AdS black holes, Phys. Rev. D 85 (2012) 024017 [arXiv:1104.3066] [INSPIRE].

[5] S. Fernando, Thermodynamics of Born-Infeld-Anti-de Sitter black holes in the grand canonical ensemble, Phys. Rev. D 74 (2006) 104032 [hep-th/0608040] [INSPIRE]. 
[6] T.K. Dey, S. Mukherji, S. Mukhopadhyay and S. Sarkar, Phase transitions in higher derivative gravity, JHEP 04 (2007) 014 [hep-th/0609038] [INSPIRE].

[7] T.K. Dey, S. Mukherji, S. Mukhopadhyay and S. Sarkar, Phase transitions in higher derivative gravity and gauge theory: R-charged black holes, JHEP 09 (2007) 026 [arXiv:0706.3996] [INSPIRE].

[8] D. Anninos and G. Pastras, Thermodynamics of the Maxwell-Gauss-Bonnet Anti-de Sitter black hole with higher derivative gauge corrections, JHEP 07 (2009) 030 [arXiv:0807.3478] [INSPIRE].

[9] M.B.J. Poshteh, B. Mirza and Z. Sherkatghanad, Phase transition, critical behavior and critical exponents of Myers-Perry black holes, Phys. Rev. D 88 (2013) 024005 [arXiv: 1306.4516] [INSPIRE].

[10] S.-W. Wei and Y.-X. Liu, Critical phenomena and thermodynamic geometry of charged Gauss-Bonnet AdS black holes, Phys. Rev. D 87 (2013) 044014 [arXiv:1209.1707] [INSPIRE].

[11] A. Lala, Critical phenomena in higher curvature charged AdS black holes, Adv. High Energy Phys. 2013 (2013) 918490 [arXiv: 1205.6121] [INSPIRE].

[12] Y.-D. Tsai, X.N. Wu and Y. Yang, Phase Structure of Kerr-AdS Black Hole, Phys. Rev. D 85 (2012) 044005 [arXiv: 1104.0502] [InSPIRE].

[13] R. Banerjee and D. Roychowdhury, Critical behavior of Born Infeld AdS black holes in higher dimensions, Phys. Rev. D 85 (2012) 104043 [arXiv: 1203.0118] [InSPIRE].

[14] R. Banerjee and D. Roychowdhury, Thermodynamics of phase transition in higher dimensional AdS black holes, JHEP 11 (2011) 004 [arXiv:1109.2433] [INSPIRE].

[15] R. Banerjee, S.K. Modak and S. Samanta, Second order phase transition and thermodynamic geometry in Kerr-AdS black hole, Phys. Rev. D 84 (2011) 064024 [arXiv:1005.4832] [INSPIRE].

[16] R. Banerjee, S.K. Modak and S. Samanta, Glassy phase transition and stability in black holes, Eur. Phys. J. C 70 (2010) 317 [arXiv:1002.0466] [InSPIRE].

[17] R. Banerjee, S. Ghosh and D. Roychowdhury, New type of phase transition in Reissner Nordstrom - AdS black hole and its thermodynamic geometry, Phys. Lett. B 696 (2011) 156 [arXiv: 1008.2644] [INSPIRE].

[18] B.P. Dolan, Pressure and volume in the first law of black hole thermodynamics, Class. Quant. Grav. 28 (2011) 235017 [arXiv:1106.6260] [INSPIRE].

[19] B.P. Dolan, The cosmological constant and the black hole equation of state, Class. Quant. Grav. 28 (2011) 125020 [arXiv:1008.5023] [INSPIRE].

[20] B.P. Dolan, Compressibility of rotating black holes, Phys. Rev. D 84 (2011) 127503 [arXiv: 1109.0198] [INSPIRE].

[21] B.P. Dolan, D. Kastor, D. Kubiznak, R.B. Mann and J. Traschen, Thermodynamic volumes and isoperimetric inequalities for de Sitter black holes, Phys. Rev. D 87 (2013) 104017 [arXiv: 1301.5926] [INSPIRE].

[22] E. Spallucci and A. Smailagic, Maxwell's equal area law for charged Anti-de Sitter black holes, Phys. Lett. B 723 (2013) 436 [arXiv:1305.3379] [INSPIRE]. 
[23] D. Kastor, S. Ray and J. Traschen, Enthalpy and the mechanics of AdS black holes, Class. Quant. Grav. 26 (2009) 195011 [arXiv:0904.2765] [INSPIRE].

[24] D. Kubiznak and R.B. Mann, P-V criticality of charged AdS black holes, JHEP 07 (2012) 033 [arXiv: 1205.0559] [INSPIRE].

[25] S. Gunasekaran, R.B. Mann and D. Kubiznak, Extended phase space thermodynamics for charged and rotating black holes and Born-Infeld vacuum polarization, JHEP 11 (2012) 110 [arXiv: 1208.6251] [INSPIRE].

[26] A. Belhaj, M. Chabab, H. El Moumni and M.B. Sedra, On thermodynamics of AdS black holes in arbitrary dimensions, Chin. Phys. Lett. 29 (2012) 100401 [arXiv:1210.4617] [INSPIRE].

[27] S.H. Hendi and M.H. Vahidinia, Extended phase space thermodynamics and P-V criticality of black holes with a nonlinear source, Phys. Rev. D 88 (2013) 084045 [arXiv:1212.6128] [INSPIRE].

[28] A. Belhaj, M. Chabab, H.E. Moumni, L. Medari and M.B. Sedra, The thermodynamical behaviors of Kerr-Newman AdS black holes, Chin. Phys. Lett. 30 (2013) 090402 [arXiv: 1307.7421] [INSPIRE].

[29] A. Belhaj, M. Chabab, H. El Moumni and M.B. Sedra, Critical behaviors of $3 D$ black holes with a scalar hair, arXiv:1306.2518 [INSPIRE].

[30] S. Chen, X. Liu, C. Liu and J. Jing, $P-V$ criticality of AdS black hole in $f(R)$ gravity, Chin. Phys. Lett. 30 (2013) 060401 [arXiv:1301.3234] [INSPIRE].

[31] N. Altamirano, D. Kubiznak and R.B. Mann, Reentrant phase transitions in rotating Anti-de Sitter black holes, Phys. Rev. D 88 (2013) 101502 [arXiv:1306.5756] [INSPIRE].

[32] N. Altamirano, D. Kubizňák, R.B. Mann and Z. Sherkatghanad, Kerr-AdS analogue of triple point and solid/liquid/gas phase transition, Class. Quant. Grav. 31 (2014) 042001 [arXiv: 1308.2672] [INSPIRE].

[33] R.-G. Cai, L.-M. Cao, L. Li and R.-Q. Yang, P-V criticality in the extended phase space of Gauss-Bonnet black holes in AdS space, JHEP 09 (2013) 005 [arXiv:1306.6233] [INSPIRE].

[34] W. Xu, H. Xu and L. Zhao, Gauss-Bonnet coupling constant as a free thermodynamical variable and the associated criticality, Eur. Phys. J. C 74 (2014) 2970 [arXiv:1311.3053] [INSPIRE].

[35] S. Dutta, A. Jain and R. Soni, Dyonic black hole and holography, JHEP 12 (2013) 060 [arXiv:1310.1748] [INSPIRE].

[36] D.-C. Zou, S.-J. Zhang and B. Wang, Critical behavior of Born-Infeld AdS black holes in the extended phase space thermodynamics, Phys. Rev. D 89 (2014) 044002 [arXiv:1311.7299] [INSPIRE].

[37] J.-X. Mo and W.-B. Liu, Ehrenfest scheme for P-V criticality of higher dimensional charged black holes, rotating black holes and Gauss-Bonnet AdS black holes, Phys. Rev. D 89 (2014) 084057 [arXiv: 1404.3872] [INSPIRE].

[38] D.-C. Zou, Y. Liu and B. Wang, Critical behavior of charged Gauss-Bonnet AdS black holes in the grand canonical ensemble, Phys. Rev. D 90 (2014) 044063 [arXiv:1404.5194] [INSPIRE]. 
[39] R. Zhao, H.-H. Zhao, M.-S. Ma and L.-C. Zhang, On the critical phenomena and thermodynamics of charged topological dilaton AdS black holes,

Eur. Phys. J. C 73 (2013) 2645 [arXiv: 1305.3725] [INSPIRE].

[40] H.P. Nollert, Quasinormal modes: the characteristic sound of black holes and neutron stars, Class. Quant. Grav. 16 (1999) R159.

[41] K.D. Kokkotas and B.G. Schmidt, Quasi-normal modes of stars and black holes, Living Rev. Rel. 2 (1999) 2

[42] B. Wang, Perturbations around black holes, Braz. J. Phys. 35 (2005) 1029.

[43] R.A. Konoplya and A. Zhidenko, Quasinormal modes of black holes: from astrophysics to string theory, Rev. Mod. Phys. 83 (2011) 793 [arXiv:1102.4014] [INSPIRE].

[44] P.C.W. Davies, Thermodynamics of black holes, Proc. Roy. Soc. Lond. A 353 (1977) 499 [INSPIRE].

[45] P.C.W. Davies, Thermodynamic phase transitions of Kerr-Newman black holes in de Sitter space, Class. Quant. Grav. 6 (1989) 1909 [InSPIRE].

[46] J. Jing and Q. Pan, Quasinormal modes and second order thermodynamic phase transition for Reissner-Nordstrom black hole, Phys. Lett. B 660 (2008) 13 [arXiv:0802.0043] [INSPIRE].

[47] E. Berti and V. Cardoso, Quasinormal modes and thermodynamic phase transitions, Phys. Rev. D 77 (2008) 087501 [arXiv:0802.1889] [INSPIRE].

[48] X. He, B. Wang, S. Chen, R.-G. Cai and C.-Y. Lin, Quasinormal modes in the background of charged Kaluza-Klein black hole with squashed horizons, Phys. Lett. B 665 (2008) 392 [arXiv: 0802.2449] [INSPIRE].

[49] S.S. Gubser and I. Mitra, The evolution of unstable black holes in Anti-de Sitter space, JHEP 08 (2001) 018 [hep-th/0011127] [INSPIRE].

[50] G. Koutsoumbas, S. Musiri, E. Papantonopoulos and G. Siopsis, Quasi-normal modes of electromagnetic perturbations of four-dimensional topological black holes with scalar hair, JHEP 10 (2006) 006 [hep-th/0606096] [INSPIRE].

[51] J. Shen, B. Wang, C.-Y. Lin, R.-G. Cai and R.-K. Su, The phase transition and the Quasi-Normal Modes of black holes, JHEP 07 (2007) 037 [hep-th/0703102] [INSPIRE].

[52] G. Koutsoumbas, E. Papantonopoulos and G. Siopsis, Phase transitions in charged topological-AdS black holes, JHEP 05 (2008) 107 [arXiv:0801.4921] [INSPIRE].

[53] X.-P. Rao, B. Wang and G.-H. Yang, Quasinormal modes and phase transition of black holes, Phys. Lett. B 649 (2007) 472 [arXiv:0712.0645] [INSPIRE].

[54] Y.S. Myung, Phase transition for black holes with scalar hair and topological black holes, Phys. Lett. B 663 (2008) 111 [arXiv:0801.2434] [InSPIRE].

[55] S.S. Gubser and I. Mitra, Instability of charged black holes in Anti-de Sitter space, hep-th/0009126 [INSPIRE].

[56] X. He, B. Wang, R.-G. Cai and C.-Y. Lin, Signature of the black hole phase transition in quasinormal modes, Phys. Lett. B 688 (2010) 230 [arXiv:1002.2679] [INSPIRE].

[57] R.-G. Cai, X. He, H.-F. Li and H.-Q. Zhang, Phase transitions in AdS soliton spacetime through marginally stable modes, Phys. Rev. D 84 (2011) 046001 [arXiv:1105.5000] [INSPIRE]. 
[58] Y. Liu and B. Wang, Perturbations around the AdS Born-Infeld black holes, Phys. Rev. D 85 (2012) 046011 [arXiv:1111.6729] [InSPIRE].

[59] E. Abdalla, C.E. Pellicer, J. de Oliveira and A.B. Pavan, Phase transitions and regions of stability in Reissner-Nordström holographic superconductors, Phys. Rev. D 82 (2010) 124033 [arXiv: 1010.2806] [INSPIRE].

[60] J.-M. Zhu, B. Wang and E. Abdalla, Object picture of quasinormal ringing on the background of small Schwarzschild Anti-de Sitter black holes, Phys. Rev. D 63 (2001) 124004 [hep-th/0101133] [INSPIRE].

[61] B. Wang, C. Molina and E. Abdalla, Evolving of a massless scalar field in Reissner-Nordstrom Anti-de Sitter space-times, Phys. Rev. D 63 (2001) 084001 [hep-th/0005143] [INSPIRE]. 